\title{
Radio seismology of the outer solar corona
}

\author{
T. V. Zaqarashvilii, ${ }^{1,4}$, V. N. Melnik ${ }^{2}$, A. I. Brazhenko ${ }^{3}$, M. Panchenko' ${ }^{1}$ A. A. Konovalenko ${ }^{2}$, A. V. Franzuzenko ${ }^{3}$, \\ V. V. Dorovskyy ${ }^{2}$, and H. O. Rucker ${ }^{1}$ \\ ${ }^{1}$ Space Research Institute, Austrian Academy of Sciences, Schmiedlstrasse 6, 8042 Graz, Austria \\ e-mail: teimuraz.zaqarashvili@oeaw.ac.at \\ 2 Institute of Radio Astronomy, Ukrainian Academy of Sciences of Ukraine, Chervonopraporna st. 4, 61002 Kharkiv, Ukraine \\ 3 Institute of Geophysics, Myasoedova str. 27/29, 36014 Poltava,Ukraine \\ 4 Abastumani Astrophysical Observatory at Ilia State University, Cholokashvili Ave. 3/5, Tbilisi, Georgia
}

Received 22 March 2013 / Accepted 8 May 2013

\begin{abstract}
Context. Observed oscillations of coronal loops in extreme ultraviolet (EUV) lines have been successfully used to estimate plasma parameters in the inner corona $\left(<0.2 R_{0}\right.$, where $R_{0}$ is the solar radius). However, coronal seismology in EUV lines fails for higher altitudes because of rapid decrease in line intensity.

Aims. We aim to use radio observations to estimate the plasma parameters of the outer solar corona $\left(>0.2 R_{0}\right)$.

Methods. We used the large Ukrainian radio telescope URAN-2 to observe type IV radio bursts at the frequency range of 8-32 MHz during the time interval of 09:50-12:30 UT on April 14, 2011. The burst was connected to C2.3 flare, which occurred in AR 11190 during 09:38-09:49 UT. The dynamic spectrum of radio emission shows clear quasi-periodic variations in the emission intensity at almost all frequencies.

Results. Wavelet analysis at four different frequencies (29 MHz, $25 \mathrm{MHz}, 22 \mathrm{MHz}$, and $14 \mathrm{MHz}$ ) shows the quasi-periodic variation of emission intensity with periods of $\sim 34 \mathrm{~min}$ and $\sim 23 \mathrm{~min}$. The periodic variations can be explained by the first and second harmonics of vertical kink oscillation of transequatorial coronal loops, which were excited by the same flare. The apex of transequatorial loops may reach up to $1.2 R_{0}$ altitude. We derive and solve the dispersion relation of trapped magnetohydrodynamic oscillations in a longitudinally inhomogeneous magnetic slab. The analysis shows that a thin (with width to length ratio of 0.1 ), dense (with the ratio of internal and external densities of $\geq 20$ ) magnetic slab with weak longitudinal inhomogeneity of the Alfvén speed may trap the observed oscillations. Seismologically estimated Alfvén speed inside the loop at the height of $\sim 1 R_{0}$ is $\sim 1000 \mathrm{~km} \mathrm{~s}^{-1}$. The magnetic field strength at this height is estimated as $\sim 0.9 \mathrm{G}$. Extrapolation of magnetic field strength to the inner corona gives $\sim 10 \mathrm{G}$ at the height of $0.1 R_{0}$.

Conclusions. Radio observations can be successfully used for the sounding of the outer solar corona, where EUV observations of coronal loops fail. Therefore, radio seismology of the outer solar corona is complementary to EUV seismology of the inner corona.
\end{abstract}

Key words. Sun: corona - Sun: oscillations - Sun: radio radiation

\section{Introduction}

Magnetohydrodynamic (MHD) waves and oscillations are ubiquitous in the solar corona (Nakariakov \& Verwichte 2005). The waves may heat the ambient plasma and accelerate solar wind particles (Aschwanden 2004). Observations show the existence of almost all MHD modes (fast kink, fast sausage, and slow magneto-acoustic waves) in solar corona (Aschwanden et al. 1999; Nakariakov et al. 1999, 2003; Ofman et al. 1999; Wang et al. 2003a,b; Wang \& Solanki 2004; Srivastava et al. 2008). Oscillations are mainly observed through imaging observations, but their spectroscopical indications are also possible. For example, purely incompressible torsional oscillation can be observed in coronal loops as periodic variation of spectral line broadening (Zaqarashvili 2003).

Observed MHD oscillations can be used to estimate plasma parameters in the solar corona using known theoretical properties of MHD waves (Roberts et al. 1984). This method, known as coronal seismology (Nakariakov \& Ofman 2001), was effectively used to determine magnetic field strength, stratification and other plasma properties of coronal loops (Arregui et al. 2007; Van Doorsselaere et al. 2007; McEwan et al. 2008; Andries et al. 2009; Verth et al. 2010; Arregui \& Asensio Ramos 2011), spicules (Zaqarashvili et al. 2007; Zaqarashvili \& Erdélyi 2009; Verth et al. 2011), and prominences (Arregui et al. 2012,
Table 1. Oscillation periods at different frequencies of radio emission obtained by wavelet analysis.

\begin{tabular}{llll}
\hline \hline $29 \mathrm{MHz}$ & $25 \mathrm{MHz}$ & $22 \mathrm{MHz}$ & $14 \mathrm{MHz}$ \\
\hline $47 \mathrm{~min}(?)$ & $40 \mathrm{~min}(?)$ & & \\
$33.5 \mathrm{~min}$ & $34 \mathrm{~min}$ & $31.5 \mathrm{~min}$ & 34.6 \\
& $23.4 \mathrm{~min}$ & $23 \mathrm{~min}$ & \\
\hline
\end{tabular}

and references therein). The oscillations are mainly observed in the inner corona, where intensity of extreme ultraviolet (EUV) lines allows us to detect active region coronal loops. In the outer corona, the oscillations are not yet detected because of EUV line intensity decrease with altitude.

Besides the EUV part of spectrum, the solar corona can be observed in the radio frequency band, which is very important for studying the rapid oscillations of the solar atmosphere owing to high temporal resolution. High-frequency oscillations $(<1 \mathrm{~min})$ are frequently detected in the radio observations (Aschwanden et al. 2004, see Table 1 in the paper). According to Aschwanden et al. (2004) most of the reported oscillations fall in the frequency range of $100-1000 \mathrm{MHz}$ corresponding to the electron densities of $10^{8}-10^{10} \mathrm{~cm}^{-3}$. Therefore, the radio emission originates from the inner corona and consequently 
the oscillations can be interpreted as fast sausage modes in short low-laying coronal loops (Nakariakov et al. 2003; Aschwanden et al. 2004). Long-period modulation (with 5-10 min) in the microwave range of $>1 \mathrm{GHz}$ can be explained by the kink oscillations of coronal loops (Khodachenko et al. 2011). Recent highresolution data obtained with the Nobeyama Radioheliograph show the frequency drift of 3-min oscillations, which can be explained by the dispersive evolution of upward propagating wave pulses (Sych et al. 2012). Hence, the radio observations are very important for studying the plasma properties in the inner corona.

On the other hand, radio observations in the frequency range of $10-100 \mathrm{MHz}$ are very important in the outer corona. Flare related activity at decameter wavelengths is manifested in a great variety of bursts: type II-IV bursts, drifting pairs, S-bursts, spikes bursts in absorption, etc. (Melnik et al. 2010). Some of them are continuations of the same bursts from decimeter and meter bands such as type III bursts, type II bursts, type IV bursts, and spikes. There are some bursts that are observed in the long meter and decameter range only, namely type IIIb bursts, drifting pairs, and S-bursts. A plasma mechanism of radio emission is usually used to explain these bursts (Ginzburg \& Zhelezniakov 1958). This mechanism has two stages. The first stage is the generation of Langmuir waves by fast electrons and the second stage is the emission of electromagnetic waves by the Langmuir waves. In this mechanism, the frequency of radio emission equals the local plasma frequency or double local frequency. So the radio emission at a given frequency originates in the plasma at a corresponding height above the solar surface. In this paper we use the decameter band $(8-32 \mathrm{MHz})$; therefore, the radio emission at these frequencies is generated at heights $\geq 1 R_{0}$, where $R_{0}$ is the solar radius, depending on the density structure of the solar corona. Type IV bursts at decameter wavelength were first observed by the radio telescope UTR-2 during the 2002-2004 observational campaign (Melnik et al. 2008). The duration of the bursts was from $1.5 \mathrm{~h}$ to several hours and fluxes were $10-10^{3}$ s.f.u. Decameter type IV bursts were usually accompanied by coronal mass ejections (CMEs) but sometimes there were no visible CMEs during type IV bursts. Specific features of decameter type IV bursts were fine structures in the form of fiber-bursts and oscillations of radio emission intensity. The largest periods of these oscillations were tens of minutes. It was suggested that the radio emission of type IV bursts originates either in coronal loops disturbed by CMEs or in the body of CMEs themselves. Hence, the observed oscillations can be connected either with oscillations of CMEs or coronal loops. Oscillation in the intensity of a type IV bursts without the associated CME is probably connected to the coronal loop oscillation triggered by a solar flare. So, the analysis of these oscillations allows us to estimate the plasma properties in the coronal loops. Among decameter bursts there are so-called inverted-U and J bursts generated by fast electron beams that propagate in the coronal loops (Dorovsky et al. 2010). Active region coronal loops may expand up to helmet streamers owing to solar wind. Additionally, very long transequatorial loops, which connect the active regions in opposite hemispheres, may reach up to the height of one solar radius. Chase et al. (1976) presented the first observational indication of transequatorial loops. Later, Pevtsov (2000) presented 87 transequatorial loops through the analysis of Yohkoh data set between 1991 October and 1998 December. The radio emission at $10-30 \mathrm{MHz}$ originate from the heights, where transequatorial and/or active region loops are located. So, the long-period modulation of emission intensity at these frequencies can be caused by the oscillations of the loops.
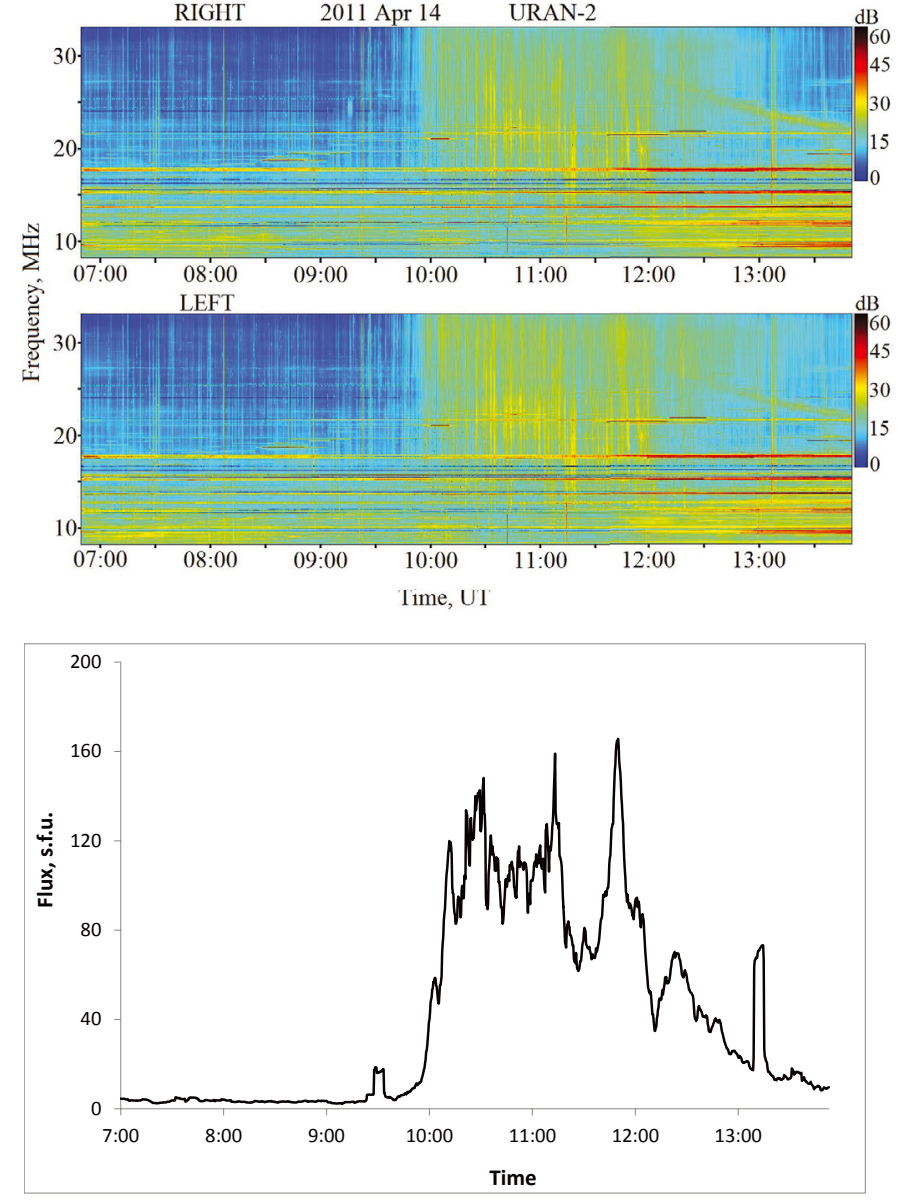

Fig. 1. Dynamic spectrum of radio emission (right and left polarization) observed by URAN-2 on April 14, 2011 (upper panel), and time profile of this radio emission at $29.7 \mathrm{MHz}$ (lower panel).

Here we use the observation of type IV radio burst from the large Ukrainian radio telescope URAN-2 for the seismological estimation of magnetic field strength and other plasma parameters of coronal loops at the heights of $\geq 0.2 R_{0}$.

\section{Observations}

The Ukrainian radio telescope URAN-2 (Poltava, Ukraine) operates in the frequency range of $8-32 \mathrm{MHz}$; it has an effective square of $28000 \mathrm{~m}^{2}$ and its beam angular resolution is $3.5^{\circ}-7^{\circ}$ (Megn et al. 2003; Brazhenko et al. 2005). The radio telescope URAN-2 can measure polarization of radio emission. The digital spectrometers DSPz (Ryabov et al. 2010) registered the solar data in the entire frequency range mentioned above. A type IV radio burst was detected in the frequency range of $\sim 12-32 \mathrm{MHz}$ during the time interval of 09:50-12:30 UT on April 14, 2011, with the frequency resolution of $4 \mathrm{kHz}$ and the time resolution of $100 \mathrm{~ms}$. Figure 1 shows the dynamic spectrum of the solar radio emission observed by radio telescope URAN-2 on April 14, 2011 , and the time profile of the radio emission at the frequency of $29.7 \mathrm{MHz}$. A decameter type IV burst started at 10:00, continued about $4 \mathrm{~h}$ and finished approximately at 14:00. The polarization of the type IV burst was high, about 40-50\%. The type IV radio burst was probably connected to a small C2.3 flare, which occurred in the active region AR 11190. The flare 


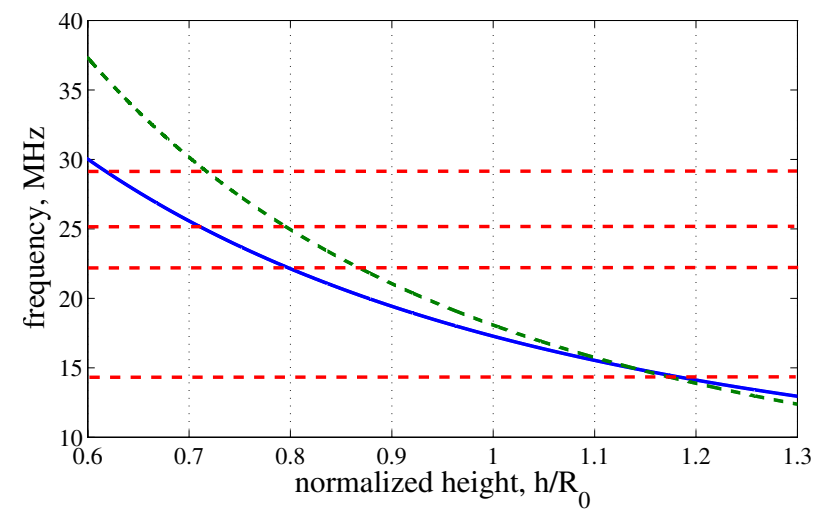

Fig. 2. Frequency of radio emission vs. height above the solar surface. The blue solid line shows the dependence according to the BaumbachAllen formula of the quiet Sun density structure; the green dashed line shows the dependence according to the gravitationally stratified atmosphere with $1 \mathrm{MK}$ temperature; the red dashed lines correspond to the frequencies $29 \mathrm{MHz}, 25 \mathrm{MHz}, 22 \mathrm{MHz}$, and $14 \mathrm{MHz}$, from top to bottom.

started at 09:38 UT, reached the maximum phase at 09:44 UT, and ended at 09:49 UT.

Type IV Radio bursts are generally produced by an emission mechanism near the plasma frequency, which depends on the electron density $n_{\mathrm{e}}$ as

$v_{\mathrm{p}}=8980 \sqrt{n_{\mathrm{e}}}$,

where $v_{\mathrm{p}}$ is in $\mathrm{Hz}$ and $n_{\mathrm{e}}$ is in $\mathrm{cm}^{-3}$. Therefore, the radio emission at a particular frequency is excited at a particular local density of emitted plasma. The density of the solar corona generally decreases with distance, hence the radio emission at different frequencies corresponds to different heights above the solar surface. The density structure of the quiet Sun corona can be approximated by the Baumbach-Allen formula (Aschwanden 2004)

$n_{\mathrm{e}}(\zeta)=10^{8}\left(\frac{2.99}{(1+\zeta)^{16}}+\frac{1.55}{(1+\zeta)^{6}}+\frac{0.036}{(1+\zeta)^{1.5}}\right) \mathrm{cm}^{-3}$,

where $\zeta=h / R_{0}$ is the normalized height above the solar surface. However, the density stratification can be different in coronal loops, which are denser than the ambient plasma. Therefore, the Baumbach-Allen formula cannot be directly applied to the coronal loops.

Figure 2 shows the radio emission frequency vs. height above the solar surface according to the Baumbach-Allen formula (Eq. (2)) and to the formula of the gravitationally stratified coronal loop (see Appendix A)

$n_{\mathrm{e}}=n_{\mathrm{e} 0} \exp \left(-\frac{R_{0}}{H_{\mathrm{n}}} \frac{\zeta}{1+\zeta}\right) \mathrm{cm}^{-3}$,

where $H_{n}=2 k T / m g$ is the density scale height near the solar surface (here $k=1.38 \times 10^{-16} \mathrm{erg} \mathrm{K}^{-1}$ is the Boltzmann constant, $m=1.67 \times 10^{-24} \mathrm{~g}$ is the proton mass, $g=2.74 \times 10^{4} \mathrm{~cm} \mathrm{~s}^{-2}$ is the gravitational acceleration near the solar surface, and $T=1 \mathrm{MK}$ is the plasma temperature) and $n_{\mathrm{e} 0}$ is chosen in such way that it is 3 times higher than the corresponding Baumbach-Allen value at the height of $0.1 R_{0}$. The curves corresponding to the Baumbach-Allen formula and gravitationally stratified corona are significantly different at lower heights, but become comparable near $1 R_{0}$. However, the two approaches may give quite different results. From this figure it is seen that the radio emission at 13-32 MHz frequency corresponds to the heights of $0.6-1.2 R_{0}$ in the case of the Baumbach-Allen formula and to the heights of $0.7-1.2 R_{0}$ in the case of gravitationally stratified corona.

\section{Quasi-periodic variations in radio emission intensity}

The dynamic spectrum of radio emission (Fig. 1) shows that the variation of emission intensity has quasi-periodic behavior in time at all frequencies, which indicates that the physical parameters of the solar corona (density of energetic electron beams, local plasma density, magnetic field strength, and/or direction etc.) undergo quasi-periodic variations. In order to understand the reason of the variations, one needs to estimate statistically significant periods in the time series.

\subsection{Wavelet analysis}

We use wavelet analysis to study the temporal variation of emission intensity at different frequencies. Figure 3 shows the wavelet spectrum of emission intensity during the interval 09:00-13:00 UT at four different frequencies, $29 \mathrm{MHz}, 25 \mathrm{MHz}$, $22 \mathrm{MHz}$, and $14 \mathrm{MHz}$.

The power spectrum of the $29 \mathrm{MHz}$ emission shows a clear peak at 33.5 min persisted during almost the whole interval. The power spectra of the $25 \mathrm{MHz}$ and $22 \mathrm{MHz}$ emissions show peaks at $34 \pm 2 \mathrm{~min}$ and $31.5 \mathrm{~min}$ respectively. The oscillations also persist during long time intervals; however, sometimes they are outside of COI. There is an additional indication of $\sim 23$ min periodicity in both plots. It should be mentioned that the power spectra of $29 \mathrm{MHz}, 25 \mathrm{MHz}$, and $22 \mathrm{MHz}$ emissions, which correspond to heights of $0.6-0.8 R_{0}$ for the BaumbachAllen formula and to $0.7-0.9 R_{0}$ for the gravitationally stratified corona, are very similar. On the other hand, the power spectrum of $14 \mathrm{MHz}$ is different, but the periodicity near $34 \mathrm{~min}$ is still present. Therefore, the oscillations with $\sim 34$ min are present at all frequencies with a significant power. There is an indication of longer period oscillation with $\sim 45 \mathrm{~min}$ at $29 \mathrm{MHz}$ and $25 \mathrm{MHz}$, but it is at the limit of convincing significance of the wavelet power spectrum. There are shorter period oscillations which exist only within short intervals, but they have much lower significance, therefore we do not consider them here.

Table 1 summarizes the results of wavelet analysis. The period of $\sim 34 \mathrm{~min}$ has stronger probability and it is seen at all frequencies during long time intervals. The period of $\sim 23 \mathrm{~min}$ is also clearly seen at the frequencies of $25 \mathrm{MHz}$ and $22 \mathrm{MHz}$.

\subsection{Possible mechanism: coronal loop oscillations}

Quasi-periodic variations in the radio emission intensity can be caused by several mechanisms. Energetic electron beams generated during the solar flare may excite Langmuir oscillations in coronal loops, which may emit the radio emission at observed frequencies as type IV bursts. Variations of electron beam density may influence the amplitude of Langmuir oscillations and consequently the intensity of radio emission. This requires the variation of the cross sections of coronal loops where the electron beam propagates. Therefore, the oscillation of magnetic tube cross sections may lead to the quasi-periodic variation of beam density and consequently to the variation of emission intensity. Periodic variation of the angle between the magnetic field direction and the line of sight may also lead to the observed oscillation. Therefore, the transverse oscillation of coronal loops can also be considered as a possible mechanism for the observations.

It is seen in Fig. 2 that the emissions at $29 \mathrm{MHz}, 25 \mathrm{MHz}$, $22 \mathrm{MHz}$, and $14 \mathrm{MHz}$ correspond to heights of $\sim 0.6 R_{0}, \sim 0.7 R_{0}$, $\sim 0.8 R_{0}$, and $\sim 1.2 R_{0}$ heights for the Baumbach-Allen formula 

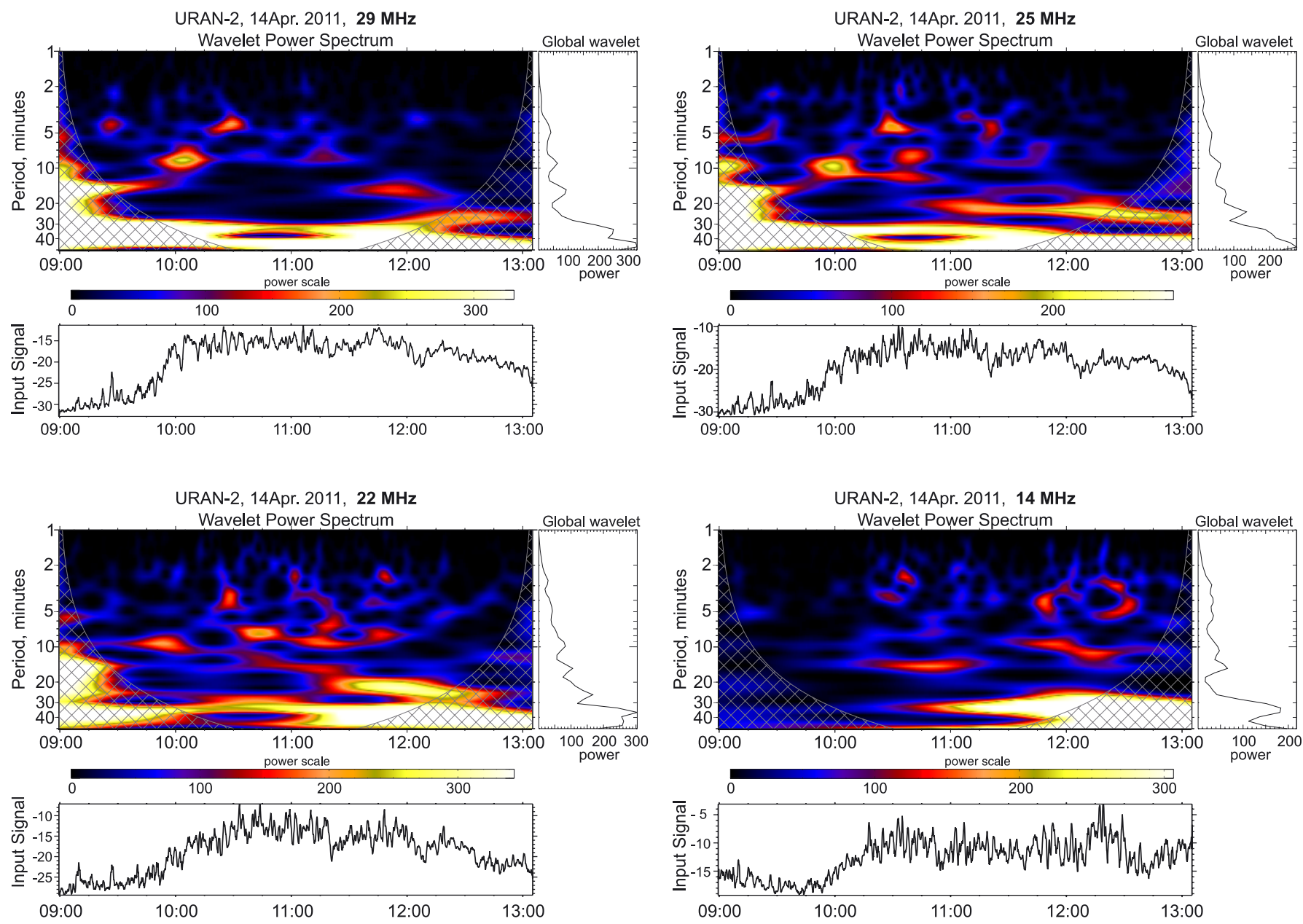

Fig. 3. Morlet wavelet power spectrum of emission intensity at $29 \mathrm{MHz}$ (upper-left panel), $25 \mathrm{MHz}$ (upper-right panel), $22 \mathrm{MHz}$ (lower left panel), and $14 \mathrm{MHz}$ (lower-right panel) during 09:00-13:00 UT. Hatched areas outside the cone of influence (COI) indicate the regions where wavelet transform is not reliable.

and $\sim 0.7 R_{0}, \sim 0.8 R_{0}, 0.9 R_{0}$, and $1.2 R_{0}$ for gravitationally stratified corona. Active region coronal loops usually reach a height of $0.1 R_{0}$ in coronal EUV lines, but in reality they may expand up to the higher values. On the other hand, very long transequatorial loops, with the length of $10^{\circ}-75^{\circ}$ heliographic degrees $\left(1^{\circ}\right.$ corresponds to $\approx 1.2 \times 10^{4} \mathrm{~km}$ ), reach greater heights (Pevtsov 2000). The apex of transequatorial loops can be located at observed heights; therefore, MHD oscillations of the loops may produce the observed variations.

A small C2.3 flare, which probably triggered the type IV radio burst, occurred in AR 11190 . Figure 4 shows the magnetogram of the solar disc at the photospheric level during the observed event obtained by the Helioseismic and Magnetic Imager (HMI, Schou et al. 2012) on board the Solar Dynamic Observatory (SDO, Pesnell et al. 2011). The active region AR 11190 is located at the central part of the northern hemisphere, while the active region AR 11187 is located just opposite in the southern hemisphere. Therefore, the two active regions could be connected by a transequatorial loop system. Transequatorial loops may connect two other active regions of the northern and southern hemispheres, namely AR 11186 and AR 11188. The distance between AR 11190 and AR 11187 is about $40^{\circ}$, which is around $\approx 4.8 \times 10^{5} \mathrm{~km}$, i.e., $0.7 R_{0}$. On the other hand, the distance between AR 11186 and AR 11188 is about $50^{\circ}$, which is around $\approx 6 \times 10^{5} \mathrm{~km}$, i.e., $0.86 R_{0}$. In the case of semi-circular shape, the transequatorial loops may reach up to $0.35 R_{0}$ in the first case and $0.43 R_{0}$ in the second case. In the case of sheared magnetic arcade, the transequatorial loops may expand farther up to the observed heights.

Figure 5 shows the solar corona in the Fe IX $171 \AA$ line during the observed events obtained by Atmospheric Imaging Assembly (AIA) on SDO (Lemen et al. 2012). The transequatorial loop system that connects the active regions AR 11190 and AR 11187 at lower heights can be seen. The loop system is probably expanded to much greater heights, but it is not seen in EUV lines.

Solar flares in AR 11190 may excite energetic electron beams along the transequatorial loops, which may trigger the Langmuir oscillations producing the observed radio emission. The solar flares may also trigger the MHD oscillations of transequatorial loops, which modulate the electron beam density and lead to the observed quasi-periodicity in radio intensity (Fig. 6).

\section{Seismology of the outer corona}

The observed oscillation periods can be used to estimate the plasma parameters of the outer solar corona using the theoretical oscillation spectrum of MHD waves in coronal loops. Analytical dispersion relations for MHD waves for straight homogeneous magnetic tubes was developed at the beginning of 1980s by Edwin \& Roberts (1983). Recent progress in coronal observations thanks to Transition Region And Coronal Explorer 


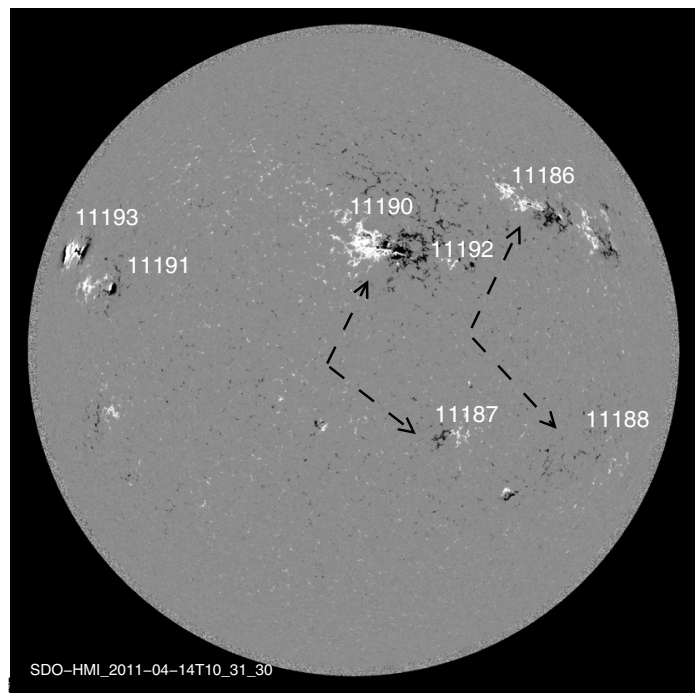

Fig. 4. Solar photospheric magnetogram at 10:31:30 UT on April 14, 2011, from SDO/HMI. Active regions are symmetrically located in northern and southern hemispheres and can be connected by transequatorial loops. The dashed arrows indicate the possibilities of transequatorial loop systems.

(TRACE) triggered numerous theoretical studies for coronal loops with longitudinal density inhomogeneity (Díaz et al. 2004; Van Doorsselaere et al. 2004; Andries et al. 2005a; Dymova \& Ruderman 2006; Donnelly et al. 2006; Zaqarashvili \& Murawski 2007), for loops with longitudinally inhomogeneous magnetic fields (Verth \& Erdélyi 2008, Pascoe et al. 2009), and for curved loops (Brady \& Arber 2005; Selwa et al. 2005, 2007; Verwichte et al. 2006; Diáz et al. 2006; Terradas et al. 2006; Gruszecki et al. 2008).

Slow magneto-acoustic oscillations can be ruled out as the reason for the observed modulation of radio emission. The fundamental standing slow wave in the coronal loop with the apex at $0.8 R_{0}$ should have a period of 3-6 $\mathrm{h}$ (for the sound speed of $150-300 \mathrm{~km} \mathrm{~s}^{-1}$ ), which is too long compared to the observed periodicity. Kink, sausage, and torsional oscillations could be responsible for the observed variation of radio emission. Kink and torsional oscillations change the direction of loop magnetic field periodically; therefore, they may lead to the variation of radio emission (Khodachenko et al. 2011). On the other hand, sausage oscillations lead to the variation of the loop cross-section and may modulate the density of electron beams, which in turn may lead to the variation of radio emission intensity. Kink oscillations in the vertical plane, known as vertical kink oscillations, may also lead to the significant variation of loop cross-sections and thus can lead to the similar effect of sausage oscillations (Aschwanden \& Schrijver 2011).

The highest altitude of emitted radio emission (corresponding to the $14 \mathrm{MHz}$ frequency) is located at $1.2 R_{0}$ (see Fig. 2). It may correspond to the apex of transequatorial coronal arcade. The mean length of the arcade can be estimated as

$$
2 L=\pi \cdot 1.2 \cdot R_{0} \approx 2.64 \times 10^{6} \mathrm{~km}
$$

for both the Baumbach-Allen formula and for the gravitationally stratified corona.

In the next sections we will use MHD oscillations of coronal loops for the estimation of plasma parameters in the outer corona using observed oscillation periods.

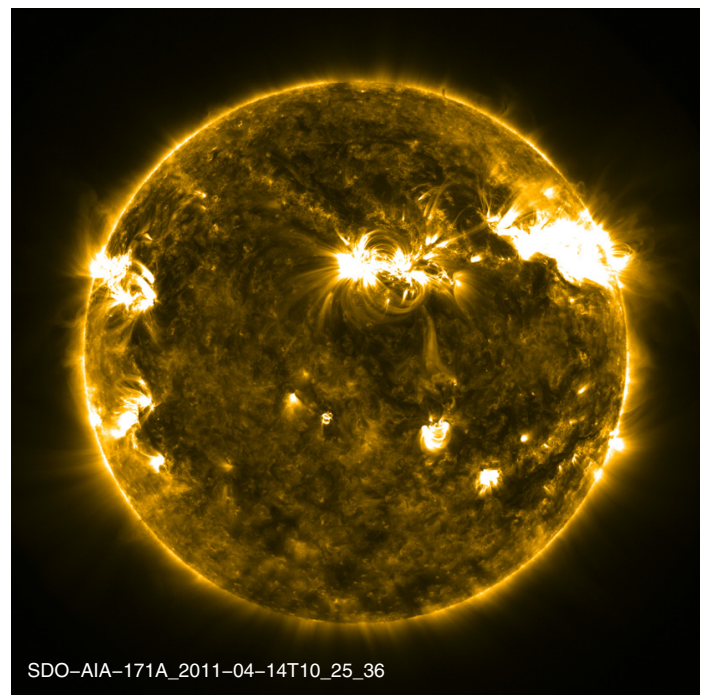

Fig. 5. Solar corona as seen in the $171 \AA$ line at 10:25:31 UT on April 14, 2011, obtained by AIA/SDO.

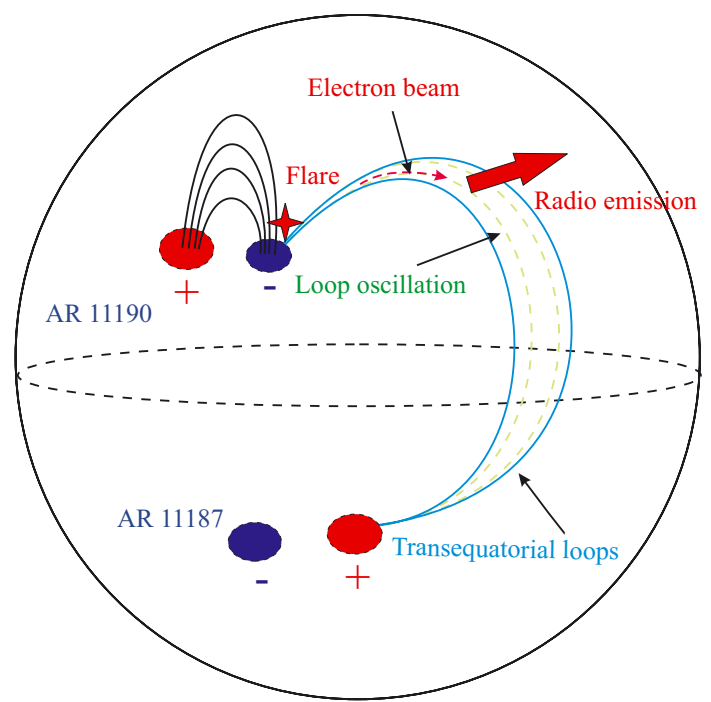

Fig. 6. Schematic picture of radio emission from a transequatorial coronal loop after a solar flare.

\subsection{Kink oscillations}

Kink waves are transverse oscillations of magnetic tubes and the phase speed for a straight homogeneous tube can be written as

$c_{\mathrm{k}}=\sqrt{\frac{\rho_{0} V_{\mathrm{A} 0}^{2}+\rho_{\mathrm{e}} V_{\mathrm{Ae}}^{2}}{\rho_{0}+\rho_{\mathrm{e}}}}=V_{\mathrm{A} 0} \sqrt{\frac{2}{1+\rho_{\mathrm{e}} / \rho_{0}}}$,

where $\rho_{0}\left(\rho_{\mathrm{e}}\right)$ is the plasma density inside (outside) the loop and $V_{\mathrm{A} 0}=B_{0} / \sqrt{4 \pi \rho_{0}}\left(V_{\mathrm{Ae}}=B_{0} / \sqrt{4 \pi \rho_{\mathrm{e}}}\right)$ is the Alfvén speed inside (outside) the loop. We note that the magnetic field strength is the same inside and outside the loop, which is a good approach in the solar corona. The horizontal transverse oscillation of coronal loops, i.e., parallel to the surface, changes the direction of the loop's magnetic field, but the density variation remains small. On the other hand, vertical kink oscillation may lead to the density variation because of the variation of loop length (Aschwanden \& Schrijver 2011).

Suppose that the observed oscillation is caused by the first harmonic of kink waves. Then the Alfvén speed inside the loop 
can be estimated as

$V_{\mathrm{A} 0}=\frac{4 L}{T_{\mathrm{obs}}} \sqrt{\frac{1+\rho_{\mathrm{e}} / \rho_{0}}{2}} \approx 2000 \mathrm{~km} \mathrm{~s}^{-1}$,

where the observed period of $T_{\mathrm{obs}}=34 \mathrm{~min}$, the loop length of $2 L=2.64 \times 10^{6} \mathrm{~km}$, and density ratio of $\rho_{\mathrm{e}} / \rho_{0}=1 / 5$ are used.

\subsection{Sausage oscillations}

Sausage waves modify the loop cross-section; therefore, they may lead to the variation of beam density and consequently the radio emission intensity. However, the long-wavelength sausage waves are evanescent in magnetic tubes (Edwin \& Roberts 1983). The expression of cut-off wave numbers can be written in the cold plasma approximation as (Roberts et al. 1984; Nakariakov et al. 2003; Aschwanden et al. 2004)

$k_{\mathrm{c}}=\frac{j_{0}}{x_{0}} \sqrt{\frac{1}{\rho_{0} / \rho_{\mathrm{e}}-1}}$,

where $x_{0}$ is the tube radius and $j_{0}=2.4$ is the first zero of the Bessel function. For the density ratio of $\rho_{0} / \rho_{\mathrm{e}}=5$, this expression leads to

$k_{\mathrm{c}} x_{0} \approx 1.2$.

The normalized wave numbers for sausage waves are

$k_{\mathrm{s}} x_{0}=\frac{2 \pi x_{0}}{4 L}(n+1), n=0,1,2 \ldots$,

where $n=0$ corresponds to the first harmonic, $n=1$ corresponds to the second, etc.

Figure 7 shows the dependence of normalized wave number $k_{\mathrm{s}} x_{0}$ of the first three harmonics on the width to length ratio (aspect ratio) of the magnetic tube. It can be seen that the first harmonic is trapped only for thick loops: the loop with density contrast $d=\rho_{0} / \rho_{\mathrm{e}}$ of 5,10 , and 20 requires the aspect ratio of $\sim 0.75, \sim 0.5$, and $\sim 0.35$, respectively. For the second harmonic the same density contrasts require $\sim 0.38, \sim 0.25$, and $\sim 0.18$. The third harmonic yields $\sim 0.25, \sim 0.18$, and $\sim 0.12$. Therefore, only the third harmonic can be trapped in reasonable values of the density contrast and aspect ratio.

The upper limit of phase speed for sausage waves is the external Alfvén speed $V_{\mathrm{Ae}}$. If one supposes that the third harmonic is responsible for the observed variation of radio emission, then the external Alfvén speed can be estimated as

$V_{\mathrm{Ae}}=\frac{4 L}{3 T_{\mathrm{obs}}} \approx 840 \mathrm{~km} \mathrm{~s}^{-1}$,

where the observed period of $T_{\mathrm{obs}}=34 \mathrm{~min}$ and the loop length of $2 L=2.64 \times 10^{6} \mathrm{~km}$ are used. The internal Alfvén speed can be estimated from the suggested density ratio of $d=\rho_{0} / \rho_{\mathrm{e}}=5$ as

$V_{\mathrm{A} 0} \approx 375 \mathrm{~km} \mathrm{~s}^{-1}$.

It is a rather small value for internal Alfvén speed at these heights.

On the other hand, if the third harmonic of sausage waves is represented by the period of $\sim 23 \mathrm{~min}$, which has significant power in the wavelet power spectrum, then the Alfvén speed outside the loop can be estimated as

$V_{\mathrm{Ae}} \approx 1275 \mathrm{~km} \mathrm{~s}^{-1}$,

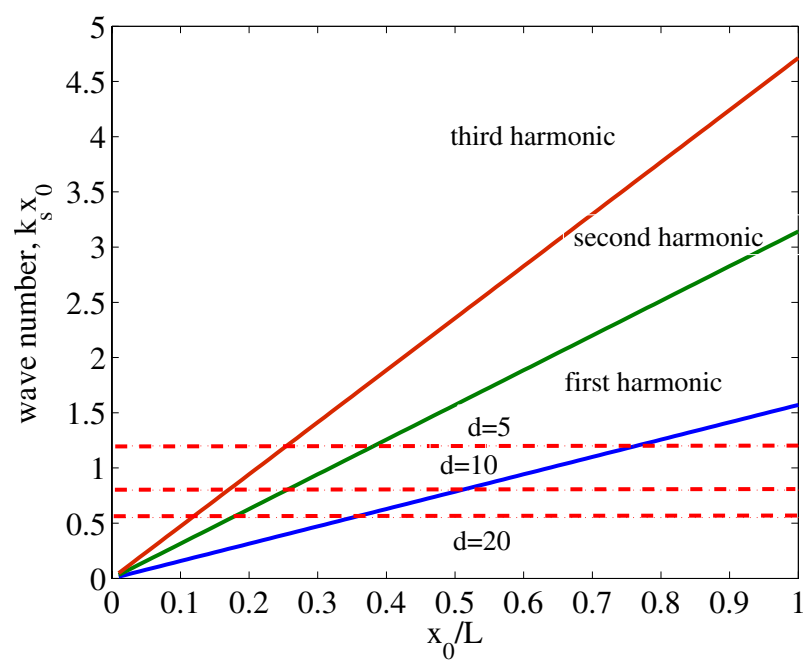

Fig. 7. Normalized wavenumber of first three harmonics of sausage waves (blue - first harmonic, green - second harmonic, dark red - third harmonic) vs. width to length ratio of the magnetic tube from Eq. (9). The red dashed lines show the cut-off wavenumbers for three different values of the ratio of internal and external densities, $d=\rho_{0} / \rho_{\mathrm{e}}=5,10$, and 20 .

which gives for the internal Alfvén speed

$V_{\mathrm{A} 0} \approx 570 \mathrm{~km} \mathrm{~s}^{-1}$

which seems to be a small value again.

The estimation of the Alfvén speed is based on the model of homogeneous coronal loops. However, the transequatorial loops are located in the higher corona, so the Alfvén speed may vary along the loop axis. Therefore, the oscillations in the loops with longitudinally inhomogeneous Alfvén speeds should be studied. Oscillations in coronal loops with step-like density profiles are well studied (Diáz et al. 2004; Dymova \& Ruderman 2006; Donnelly et al. 2006). However, it is desirable to consider a smooth profile of Alfvén speed, so we use the smooth profile adopted by Zaqarashvili \& Murawski (2007).

\subsection{Oscillations of coronal loops with longitudinally inhomogeneous Alfvén speed}

Since slow magneto-acoustic waves are probably not relevant to our observations, we implement a zero plasma- $\beta$ approximation and take into account small amplitude perturbations. We use the Cartesian coordinate system $(x, z)$, so the considered magnetic structure is a slab rather than a loop. Consideration of cylindrical magnetic tubes makes the oscillation spectrum more comprehensive, but the slab model is also a good approximation for our purposes. The equation governing the linear perturbations in the system can be written after Fourier analysis with $\exp (-\mathrm{i} \omega t)$ as

$\frac{\partial^{2} u_{x}}{\partial x^{2}}+\frac{\partial^{2} u_{x}}{\partial z^{2}}+\frac{\omega^{2}}{V_{\mathrm{A}}^{2}} u_{x}=0$,

where $u_{x}$ is the velocity perturbation, $V_{\mathrm{A}}=B_{0} / \sqrt{4 \pi \varrho}$ is the Alfvén speed, $\varrho$ is the plasma density, $B_{0}$ is the magnetic field strength, and $\omega$ is a wave frequency. Equation (14) accompanied by appropriate boundary conditions, consists of a classical boundary-value problem.

We consider a straight dense coronal loop that is embedded in a rarified plasma. The low plasma- $\beta$ condition in the solar corona implies that the enhanced hydrodynamic pressure due 
to the dense and hot plasma inside a coronal loop can be easily compensated by very small differences in the magnetic field strength inside and outside the loop. The Alfvén speed inside and outside the loop is given as

$V_{\mathrm{A} 0}\left(1+\alpha^{2} \frac{z^{2}}{L^{2}}\right)^{-1 / 2}$, for $x<x_{0}$,

$V_{\mathrm{Ae}}\left(1+\frac{\varrho_{00}}{\varrho_{0 \mathrm{e}}} \alpha^{2} \frac{z^{2}}{L^{2}}\right)^{-1 / 2}$ for $x>x_{0}$,

where $V_{\mathrm{A} 0}=B_{0} / \sqrt{4 \pi \varrho_{00}}\left(V_{\mathrm{Ae}}=B_{0} / \sqrt{4 \pi \varrho_{0 \mathrm{e}}}\right)$ is the internal (external) Alfvén speed at the loop apex $(z=0), \varrho_{00}$ and $\varrho_{0 \mathrm{e}}$ are the corresponding densities, and $\alpha$ defines the strength of the inhomogeneity. The profile states that the Alfvén speed is smaller at loop footpoints and becomes higher at the loop apex.

In order to find a dispersion relation of possible oscillation modes in the system one can find the solutions of Eq. (14) inside and outside the tube that satisfy the closed boundary conditions at the loop footpoints, and then merge the solutions at the tube boundary (Roberts 1981).

The velocity and the total pressure perturbations should be continuous at the loop boundary $x=x_{0}$,

$\left[u_{x}\right]=0,\left[P=\frac{B_{0} b_{z}}{4 \pi}\right]=0$.

Here $b_{z}$ is the $z$ component of the magnetic field perturbation, and $[f]$ indicates the jump of $f$ across the boundary. We implement line-tying conditions at loop footpoints

$u_{x}(z= \pm L)=0$.

Inside and outside the loop, Eq. (14) has the following form:

$\frac{\partial^{2} u_{x \mathrm{i}}}{\partial x^{2}}+\frac{\partial^{2} u_{x \mathrm{i}}}{\partial z^{2}}+\left[\frac{\omega^{2}}{V_{\mathrm{A} 0}^{2}}+\alpha^{2} \frac{\omega^{2}}{V_{\mathrm{A} 0}^{2}} \frac{z^{2}}{L^{2}}\right] u_{x \mathrm{i}}=0$,

$\frac{\partial^{2} u_{x \mathrm{e}}}{\partial x^{2}}+\frac{\partial^{2} u_{x \mathrm{e}}}{\partial z^{2}}+\left[\frac{\omega^{2}}{V_{\mathrm{Ae}}^{2}}+\alpha^{2} \frac{\omega^{2}}{V_{\mathrm{A} 0}^{2}} \frac{z^{2}}{L^{2}}\right] u_{x \mathrm{e}}=0$,

where $u_{x \mathrm{i}}\left(u_{x \mathrm{e}}\right)$ is the transverse velocity perturbation inside (outside) the loop.

We use the method of separation of variables in order to solve Eqs. (19) and (20). The two equations are similar, so it is enough to solve Eq. (19). Equation (20) can then be solved analogously. Setting $u_{x \mathrm{i}}(x, z)=\Phi_{\mathrm{i}}(x) \Psi_{\mathrm{i}}(z)$ we get from Eq. (19)

$\frac{\mathrm{d}^{2} \Psi_{\mathrm{i}}}{\mathrm{d} z^{2}}+\alpha^{2} \frac{\omega^{2}}{V_{\mathrm{A} 0}^{2}} \frac{z^{2}}{L^{2}} \Psi_{\mathrm{i}}=-k^{2} \Psi_{\mathrm{i}}$,

$\frac{\mathrm{d}^{2} \Phi_{\mathrm{i}}}{\mathrm{d} x^{2}}+\left[\frac{\omega^{2}}{V_{\mathrm{A} 0}^{2}}-k^{2}\right] \Phi_{\mathrm{i}}=0$,

where $k^{2}$ is the separation constant. Boundary conditions (Eq. (18)) are now rewritten as

$\Psi_{\mathrm{i}}(z= \pm L)=0$.

Equations (21) and (23) consist the well-known Sturm-Liouville problem, which requires to find the solutions of Eq. (21) that satisfy the boundary condition (Eq. (23)).

With the use of the notations (Zaqarashvili \& Murawski 2007)

$\xi \equiv \sqrt{\frac{2 \alpha \omega}{V_{\mathrm{A} 0} L}} z, \quad a \equiv-\frac{V_{\mathrm{A} 0} L}{2 \alpha \omega} k^{2}$
Equation (21) can be rewritten in the form of the Weber (parabolic cylinder) equation (Abramowitz \& Stegun 1964)

$\frac{\mathrm{d}^{2} \Psi_{\mathrm{i}}}{\mathrm{d} \xi^{2}}+\left(\frac{\xi^{2}}{4}-a\right) \Psi_{\mathrm{i}}=0$

Standard solutions to this equation are called Weber (parabolic cylinder) functions, $W(a, \pm \xi)$ (Abramowitz \& Stegun 1964)

$W(a, \pm \xi)=\frac{(\cosh \pi a)^{1 / 4}}{2 \sqrt{\pi}}\left(G_{1} y_{1}(\xi) \mp \sqrt{2} G_{3} y_{2}(\xi)\right)$,

where

$G_{1}=\left|\Gamma\left(\frac{1}{4}+\frac{\mathrm{i} a}{2}\right)\right|, \quad G_{3}=\left|\Gamma\left(\frac{3}{4}+\frac{\mathrm{i} a}{2}\right)\right|$

and $y_{1}(\xi), y_{2}(\xi)$ are, respectively, even and odd solutions to Eq. (25) such as

$y_{1}(\xi)=1+a \frac{\xi^{2}}{2 !}+\left(a^{2}-\frac{1}{2}\right) \frac{\xi^{4}}{4 !}+\cdots$,
$y_{2}(\xi)=\xi+a \frac{\xi^{3}}{3 !}+\left(a^{2}-\frac{3}{2}\right) \frac{\xi^{5}}{5 !}+\cdots$.

In the case of a weakly inhomogeneous Alfvén speed, i.e., $\alpha^{2} \ll$ 1, Eq. (25) has periodic solutions when

$a<0,-a \gg \xi^{2}, p \equiv \sqrt{-a}$.

We adopt the expansion (Abramowitz \& Stegun 1964; see also Zaqarashvili \& Murawski 2007)

$$
W(a, \xi)+\mathrm{i} W(a,-\xi)=
$$

$\sqrt{2} W(a, 0) \exp \left[v_{\mathrm{r}}+\mathrm{i}\left(p \xi+\pi / 4+v_{\mathrm{i}}\right)\right]$,

where

$$
\begin{aligned}
W(a, 0) & =\frac{1}{2^{3 / 4}} \sqrt{\frac{G_{1}}{G_{3}}}, \\
v_{\mathrm{r}} & =-\frac{(\xi / 2)^{2}}{(2 p)^{2}}+\frac{2(\xi / 2)^{4}}{(2 p)^{4}}+\cdots, \\
v_{\mathrm{i}} & =\frac{2 / 3(\xi / 2)^{3}}{2 p}+\cdots .
\end{aligned}
$$

As a result of relation $-a \gg \xi^{2}$ we have from Eq. (30)

$W(a, \xi)=\sqrt{2} W(a, 0) \exp \left(-\frac{\xi^{2}}{16 p^{2}}\right) \cos \zeta$,
$W(a,-\xi)=\sqrt{2} W(a, 0) \exp \left(-\frac{\xi^{2}}{16 p^{2}}\right) \sin \zeta$,

$\zeta \equiv p \xi+\pi / 4+\frac{\xi^{3}}{24 p}$

The general solution to Eq. (25) is

$\Psi_{\mathrm{i}}=c_{1} W(a, \xi)+c_{2} W(a,-\xi)$,

where $c_{1}$ and $c_{2}$ are constants.

Equation (37) describes a periodic function and therefore it may easily satisfy the line-tying boundary conditions of Eq. (23). It is noteworthy that there are two sets of solutions of Eq. (37). For one set there is no node at the loop apex, i.e., at $\xi=0$. These are odd solutions which imply $c_{1}=c_{2}$ and correspond to the 
first, third, etc., standing modes. For the other set there is a node at $\xi=0$ and the solutions imply $c_{1}=-c_{2}$. They correspond to the second, fourth, etc., standing modes. Both sets satisfy the boundary conditions of Eq. (23).

In the case of odd solutions, the line-tying boundary conditions of Eq. (23) determine $k_{\mathrm{n}}$,

$k_{\mathrm{n}}^{2}-\frac{(2 n+1) \pi}{2 L} k_{\mathrm{n}}+\frac{\alpha^{2}}{6} \frac{\omega^{2}}{V_{\mathrm{A} 0}^{2}}=0, \quad n=0,1,2, \cdots$.

We have

$k_{\mathrm{n}}^{2}=\frac{(2 n+1)^{2} \pi^{2}}{8 L^{2}}\left(1+\sqrt{1-\frac{\alpha^{2}}{3} \frac{\omega^{2}}{V_{\mathrm{A} 0}^{2}} \frac{8 L^{2}}{(2 n+1)^{2} \pi^{2}}}\right)-\frac{\alpha^{2}}{6} \frac{\omega^{2}}{V_{\mathrm{A} 0}^{2}}$.

Using Eq. (39) we rewrite Eq. (22) inside and outside the loop as

$\frac{\mathrm{d}^{2} \Phi_{\mathrm{i}}}{\mathrm{d} x^{2}}-m_{\mathrm{i}}^{2} \Phi_{\mathrm{i}}=0$

$\frac{\mathrm{d}^{2} \Phi_{\mathrm{e}}}{\mathrm{d} x^{2}}-m_{\mathrm{e}}^{2} \Phi_{\mathrm{e}}=0$

where

$m_{\mathrm{i}}^{2}=k_{\mathrm{n}}^{2}-\frac{\omega^{2}}{V_{\mathrm{A} 0}^{2}}$

and

$m_{\mathrm{e}}^{2}=k_{\mathrm{n}}^{2}-\frac{\omega^{2}}{V_{\mathrm{Ae}}^{2}}$

For even solutions the line-tying conditions give

$k_{\mathrm{n}}^{2}-\frac{n \pi}{L} k_{\mathrm{n}}+\frac{\alpha^{2}}{6} \frac{\omega^{2}}{V_{\mathrm{A} 0}^{2}}=0, \quad n=1,2,3, \ldots$,

which leads to

$k_{\mathrm{n}}^{2}=\frac{n^{2} \pi^{2}}{2 L^{2}}\left(1+\sqrt{1-\frac{\alpha^{2}}{3} \frac{\omega^{2}}{V_{\mathrm{A} 0}^{2}} \frac{2 L^{2}}{n^{2} \pi^{2}}}\right)-\frac{\alpha^{2}}{6} \frac{\omega^{2}}{V_{\mathrm{A} 0}^{2}}$.

The solution of transverse velocity inside the slab from Eq. (40) is

$\Phi_{\mathrm{i}}=b_{1} \cosh m_{\mathrm{i}} x+b_{2} \sinh m_{\mathrm{i}} x$,

where $b_{1}$ and $b_{2}$ are arbitrary constants. On the other hand, outside the slab the solutions are

$\Phi_{\mathrm{e}}=d_{1} \mathrm{e}^{-m_{\mathrm{e}}\left(x-x_{0}\right)}$, for $x>x_{0}$,

$\Phi_{\mathrm{e}}=d_{2} \mathrm{e}^{m_{\mathrm{e}}\left(x+x_{0}\right)}$, for $x<-x_{0}$.

The solutions outside the slab must vanish at infinity, which means that $m_{\mathrm{e}}>0$. Additionally, we consider a trapped solution, which means that the oscillations should be confined inside the slab. Therefore, we should avoid the oscillatory behavior outside the loop, i.e., leaky modes. Hence, $m_{\mathrm{e}}$ should be real value, i.e., $m_{\mathrm{e}}^{2}>0$. This condition leads to the restriction on wave frequency, which for small $\alpha$ reads as (providing that $\omega>0$ )

$\omega<\frac{(2 n+1) \pi V_{\mathrm{Ae}}}{2 L}\left(1+\frac{\alpha^{2} V_{\mathrm{Ae}}^{2}}{3 V_{\mathrm{A} 0}^{2}}\right)^{-1 / 2}$,
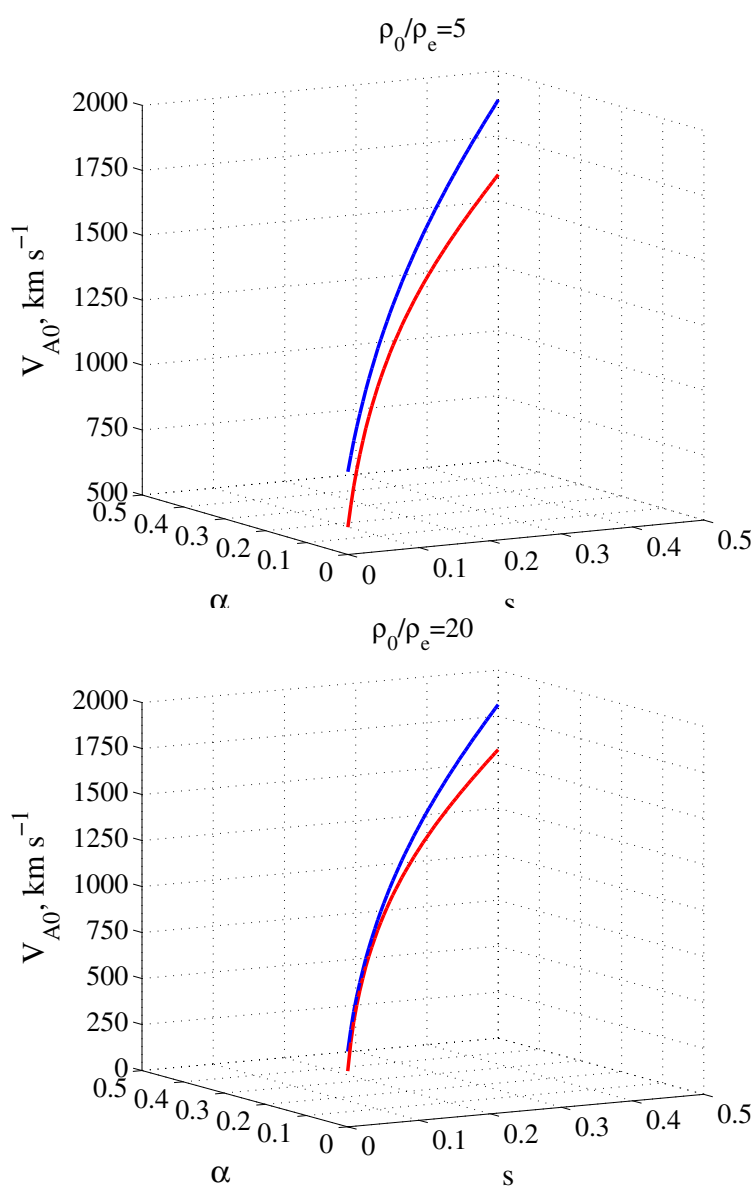

Fig. 8. Estimated Alfvén speed vs. aspect ratio $s=x_{0} / L$, and inhomogeneity parameter $\alpha$ of coronal loops. Blue (red) line corresponds to the first (second) harmonic of kink waves according to Eq. (54) (Eq. (55)). The upper panel corresponds to $\rho_{0} / \rho_{\mathrm{e}}=5$ and the lower panel to $\rho_{0} / \rho_{\mathrm{e}}=20$. Only one line from each surface of $V_{\mathrm{A} 0}(s, \alpha)$ is shown here.

for the odd modes and

$\omega<\frac{n \pi V_{\mathrm{Ae}}}{L}\left(1+\frac{\alpha^{2} V_{\mathrm{Ae}}^{2}}{3 V_{\mathrm{A} 0}^{2}}\right)^{-1 / 2}$

for even modes.

To obtain the dispersion relation, we need the expression of total pressure perturbations which is readily obtained

$P=\frac{B_{0} b_{z}}{4 \pi}=-\mathrm{i} \frac{B_{0}^{2}}{4 \pi \omega} \frac{\partial u_{x}}{\partial x}$.

Continuity of transverse velocity and total pressure perturbations at slab boundaries $x= \pm x_{0}$ gives the dispersion relations for even modes (with respect to $x$ )

$m_{\mathrm{i}} \sinh m_{\mathrm{i}} x_{0}+m_{\mathrm{e}} \cosh m_{\mathrm{i}} x_{0}=0$,

and odd modes (with respect to $x$ )

$m_{\mathrm{i}} \cosh m_{\mathrm{i}} x_{0}+m_{\mathrm{e}} \sinh m_{\mathrm{i}} x_{0}=0$.

The odd mode has a velocity node at the tube center $x=0$ and is equivalent to sausage waves, while the even mode has no velocity node at $x=0$ and is equivalent to kink waves. Kink waves lead to the transverse displacement of the slab axis, while sausage modes do not (Roberts 1981). 


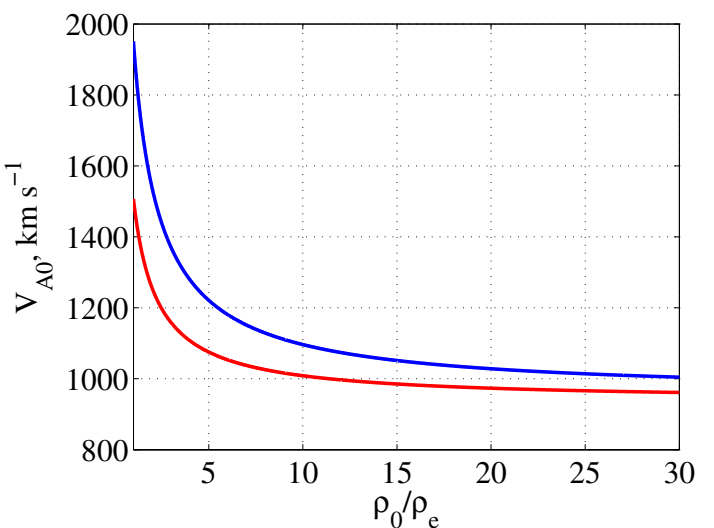

Fig. 9. Estimated Alfvén speed vs. density contrast. Blue and red lines correspond to the Alfvén speeds estimated from the first and the second harmonics of kink waves, respectively.

\subsection{Estimation of plasma parameters in the outer solar corona}

Equation (52) can be solved analytically for $m_{\mathrm{i}} x_{0} \ll 1$, which yields $\sinh m_{\mathrm{i}} x_{0} \approx m_{\mathrm{i}} x_{0}$ and $\cosh m_{\mathrm{i}} x_{0} \approx 1$. Then the frequency of the first harmonic of kink waves can be approximated as (assuming $s, \alpha$, and $\rho_{\mathrm{e}} / \rho_{0}$ to be small)

$\omega_{1 \mathrm{k}} \approx \sqrt{\frac{2+\pi s}{\pi\left(1+\alpha^{2} / 3\right) s+\rho_{\mathrm{e}} / \rho_{0}}} \frac{\pi V_{\mathrm{A} 0}}{2 L}$,

where $s=x_{0} / L$ is the ratio of loop width and length, while the frequency of the second harmonic is

$\omega_{2 \mathrm{k}} \approx \sqrt{\frac{2(1+\pi s)}{2 \pi\left(1+\alpha^{2} / 3\right) s+\rho_{\mathrm{e}} / \rho_{0}}} \frac{\pi V_{\mathrm{A} 0}}{L}$.

If the observed periods of radio emission intensity variation with $34 \mathrm{~min}$ and $23 \mathrm{~min}$ correspond to the first and second harmonics of kink waves, then Eqs. (54) and (55) allow us to estimate the value of Alfvén speed $V_{\mathrm{A} 0}$ as well as the values of $s$ and $\alpha$. The Alfvén speed should be the same as estimated from both harmonics, therefore we should find the values of $s$ and $\alpha$, in which the condition is satisfied. Figure 8 shows that the curves corresponding to the estimated Alfvén speed for the first and second harmonics do not intersect in the case of $\rho_{0} / \rho_{\mathrm{e}}=5$ (here we use the loop length of $2 L=2.64 \times 10^{6} \mathrm{~km}$ ). Therefore, the observations cannot be explained by kink oscillations in this case. On the other hand, the curves approach each other for $\rho_{0} / \rho_{\mathrm{e}}=20$ if

$s=\frac{x_{0}}{L} \approx 0.05-0.1$

and

$\alpha \approx 0.05-0.1$.

In this case, the Alfvén speed in the intersection region $(\alpha=0.1$, $s=0.1)$ is of the order of

$V_{\mathrm{A} 0} \approx 1000 \mathrm{~km} \mathrm{~s}^{-1}$.

Figure 9 shows the estimated Alfvén speed vs. the density contrast for $\alpha=0.1$ and $s=0.1$ according to Eqs. (54) and (55). It is clearly seen that the Alfvén speeds estimated from the first and the second harmonics are very different for small density contrasts, but become relatively similar for higher density contrasts and tend to $\sim 1000 \mathrm{~km} \mathrm{~s}^{-1}$.

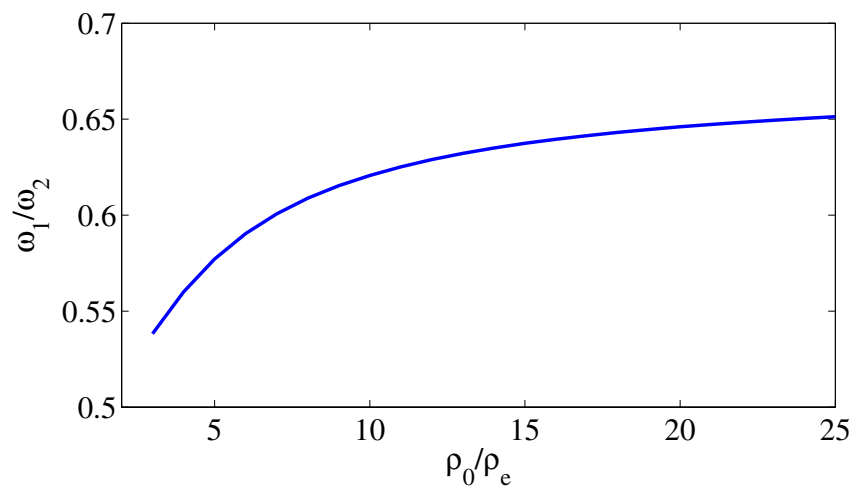

Fig. 10. Ratio of frequencies of first and second harmonics vs. the ratio of internal to external densities. The aspect ratio is taken as $x_{0} / L=0.1$ and the inhomogeneity parameter as $\alpha=0.1$.

We solved the dispersion relation for kink waves (Eq. (52)) numerically. The ratio of the frequencies of first and second harmonics vs. the ratio of internal to external densities is plotted in Fig. 10. The ratio increases for large $\rho_{0} / \rho_{\mathrm{e}}$. On the other hand, the ratio of observed oscillation periods is $23 / 35 \sim 0.65$. Therefore, it can be seen that $\rho_{0} / \rho_{\mathrm{e}}$ should be at least $\geq 20$ in order to explain the observations. This means that the transequatorial loops are much denser than the surrounding plasma in the outer corona. However, this estimation was derived on the base of calculations for a straight coronal slab. Consideration of a more realistic situation of curved loop with the tube geometry may significantly change the estimation.

The value of Alfvén speed (Eq. (58)) is estimated near the height of $1 R_{0}$ which is the averaged altitude of the transequatorial loop apex. Then the Alfvén speed at the coronal base can be estimated from Eq. (15) for $\alpha=0.1$ as

$V_{\mathrm{A} 0} \approx 995 \mathrm{~km} \mathrm{~s}^{-1}$.

Therefore, the Alfvén speed stays almost unchanged from the coronal base up to the height of $\sim R_{0}$.

Alfvén speed and electron number density allow us to estimate the magnetic field strength inside the loop in the outer corona. Electron number density can be obtained from the observed radio frequencies according to Eq. (1) (the radio frequencies of 30-14 MHz correspond to the averaged value of electron number density of $4 \times 10^{6} \mathrm{~cm}^{-3}$ ). We note that the same value is estimated from gravitationally stratified corona at the height of $1 R_{0}$. Then, Eq. (58) gives the value of mean magnetic field strength as

$B_{0} \approx 0.9 \mathrm{G}$.

\section{Discussion}

Observed oscillations are important tools to estimating the plasma parameters in the solar atmosphere. In the case of coronal loops, the observed oscillations in EUV line intensity may allow us to estimate the magnetic field strength in the corona up to $0.1 R_{0}$. In other cases, the oscillations in the $\mathrm{H} \alpha$ line may allow us to estimate the magnetic field strength in chromospheric spicules and prominences. The coronal loops are not seen in the outer corona $\left(>0.2 R_{0}\right)$ because of the decrease in EUV intensity. Therefore, the coronal seismology does not allow us to estimate the plasma parameters in the outer corona. On the other hand, radio emissions at the frequency range of $10-100 \mathrm{MHz}$, which corresponds to the heights of $0.2-1.3 R_{0}$, can be successfully used for the radio sounding of these heights. 
We analyzed the type IV radio burst at the frequency range of $13-32 \mathrm{MHz}$ which corresponds to the heights of $0.6-1.3 R_{0}$ as estimated from the Baumbach-Allen formula and to the heights of $0.7-1.2 R_{0}$ as estimated from the gravitationally stratified corona for $1 \mathrm{MK}$ temperature (Fig. 2). The type IV radio burst was connected to small C2.3 flare. The burst is believed to be caused by plasma mechanism of radio emission from Langmuir waves, which are triggered by energetic electron beams after the solar flare. The electrons are accelerated along the coronal loops, therefore the radio emission probably originates from the loops. There are two possible kinds of loops, which may expand up to the observed heights. The active region loops, which connect the opposite polarities in the same active region, may expand up to higher corona. Consequently the closed loops may reach the base of helmet streamers, where the magnetic field lines start to open. On the other hand, the transequatorial loops, which connect the active regions of opposite hemispheres, may reach the observed heights, because of large distance between their footpoints. At least two different transequatorial loop systems may exist on the visible part of the Sun during the observed type IV burst (see Fig. 4). One of them is connected to the active region where the flare occurred. Therefore we suggest that the observed radio emission was originated from the transequatorial loop system, which connects the active regions AR 11190 and AR 11187 (see Fig. 6).

We applied the wavelet analysis to study the periodic variations of type IV burst intensity at four frequencies: $29 \mathrm{MHz}$, $25 \mathrm{MHz}, 22 \mathrm{MHz}$, and $14 \mathrm{MHz}$. The analysis shows two clear periodicities in the emission intensity with significant probability (Fig. 3). The period of $\sim 34$ min has stronger peak in the power spectrum and it appears at all frequencies of radio emission. The period of $\sim 23$ min appears only at frequencies of $25 \mathrm{MHz}$ and $22 \mathrm{MHz}$ and it is absent at frequencies of $29 \mathrm{MHz}$ and $14 \mathrm{MHz}$. We suggest that the periodicity in the emission intensity can be caused by MHD oscillations of coronal loops, which were triggered by the same flare. The apex of the coronal loop is located at the height of $1.2 R_{0}$ using the estimations from both, the Baumbach-Allen formula (Eq. (2)), and the gravitationally stratified atmosphere with the temperature of $1 \mathrm{MK}$ (Eq. (3)). The possible length of the loop and, consequently, the phase speed of 34 min oscillations can be estimated to be $2.64 \times 10^{6} \mathrm{~km}$ and $2500 \mathrm{~km} \mathrm{~s}^{-1}$, respectively, if the fundamental harmonic is concerned. Therefore, we may rule out the slow magneto-acoustic mode as the reason of the periodicity because of its low phase speed. There are still three MHD modes, which could be responsible for the oscillations: kink, sausage, and torsional Alfvén modes. The sausage waves have long wavelengths cut-off in magnetic tubes, so that the first and second harmonics seem to be evanescent in typical coronal loops. The third harmonic can be trapped in some conditions; however, the estimation gives a rather small value of the Alfvén speed in the corona (see Sect. 4.2). Therefore, we think that the sausage waves can be also ruled out. It is hard to excite torsional oscillations in coronal loops, therefore fast kink waves are the best candidates for explaining the observed periodicity. There are two types of kink modes in coronal loops, the horizontal kink mode, which displaces the loop horizontally, and the vertical kink mode, which displaces the loop vertically. Horizontal kink oscillations are frequently observed in the solar corona. They are almost incompressible, hence do not lead to significant variation in plasma density. The oscillations damp quickly over a few wave periods. On the other hand, vertical kink oscillations are more rarely observed. Recently, Aschwanden \& Schrijver (2011) found a very interesting example of vertical kink oscillations. The oscillations were accompanied by variations of cross section/density and they showed no damping during the interval of four oscillation periods. They suggested that the variation of density and cross section is the result of the variation of loop length during the oscillation. In our case, the oscillation is accompanied by variations of cross section (because it should modulate the electron beam density) and it does not show the damping during several wave periods. Therefore, we think that the oscillation, which resulted in the variation of radio emission intensity in a type IV burst, has properties similar to those reported by Aschwanden \& Schrijver (2011), hence it is the vertical kink oscillation of the transequatorial loop.

Observation of two different periods in the emission intensity ( $34 \mathrm{~min}$ and $23 \mathrm{~min}$ ) can be explained by the first and second harmonics of standing oscillations. Their ratio is $\sim 0.67$ significantly shifted from 0.5 , which may cause some problems at first glance. However, the ratio in the magnetic tubes indeed is different than 0.5 . There are several observational examples of simultaneous existence of first and second harmonics of kink oscillations (Van Doorsselaere et al. 2004; Verwichte et al. 2004; De Moortel \& Brady 2007) and sausage oscillations (Srivastava et al. 2008) in coronal loops. The ratio between the frequencies of the first and second harmonics was significantly shifted from 0.5 in all observations, which was explained as the result of longitudinal density stratification in the loop (Andries et al. 2005b; McEwan et al. 2006). It should be mentioned, however, that the finite radius of the tube and the density contrast inside and outside the tube also lead to the shift of the ratio from 0.5 . We solved the MHD equations for a coronal slab with longitudinally inhomogeneous Alfvén speed and derived the dispersion relation for kink and sausage waves. From the observed frequency ratio we then found that the value of the inhomogeneity parameter $\alpha$ and the aspect ratio (the ratio of loop width and length) should be around 0.1 , while the density contrast should be large enough, $\rho_{0} / \rho_{\mathrm{e}}>20$. Therefore, the analysis is in favor for a dense loop with weak longitudinal inhomogeneity of the Alfvén speed. However, the analysis was performed for a straight coronal loop with the slab geometry. On the other hand, more real situation of curved coronal loop with the tube geometry may significantly change the situation.

The Alfvén speed inside the loop at its apex (approximately $1 R_{0}$ ) for $\alpha=0.1, x_{0} / L=0.1$ and $\rho_{0} / \rho_{\mathrm{e}}=20$ is estimated as $\sim 1000 \mathrm{~km} \mathrm{~s}^{-1}$. Then the magnetic field strength inside the loop can be derived fixing the value of the loop density. The electron number density inside the loop can be obtained from observed radio frequencies of $14-29 \mathrm{MHz}$ which correspond to the averaged value of electron number density of $4 \times 10^{6} \mathrm{~cm}^{-3}$. We note that the same value is estimated from the gravitationally stratified corona with $1 \mathrm{MK}$ temperature at the height of $1 R_{0}$. Using the value of loop density we may estimate the mean magnetic field strength at the height of $1 R_{0}$ to be $\sim 0.9 \mathrm{G}$. We may then extrapolate the Alfvén speed towards the loop footpoints using $\alpha=0.1$ and it gives $\sim 995 \mathrm{~km} \mathrm{~s}^{-1}$. Therefore, the Alfvén speed does not significantly vary with height up to 1 solar radius, hence it yields $B(z) \sim \sqrt{\rho(z)}$. However, the estimated density contrast $\rho_{0} / \rho_{\mathrm{e}}=20$ seems to be high at these heights. For the electron number density of $4 \times 10^{6} \mathrm{~cm}^{-3}$ inside the loop (estimated from radio emission frequencies), the density contrast leads to the electron number density outside the loop as $2 \times 10^{5} \mathrm{~cm}^{-3}$. On the other hand, the Baumbach-Allen formula estimates the electron number density outside the active region to be $\sim 3.7 \times 10^{6} \mathrm{~cm}^{-3}$ at $1 R_{0}=1$, which is much higher than the estimated value. The requested value of external density can be obtained from the Baumbach-Allen formula only near $5 R_{0}$, which seems to be 
a much greater height for coronal loops. Therefore, either the Baumbach-Allen formula is not valid at the heights of $1 R_{0}$, or the estimations require further revision. The Baumbach-Allen formula has been tested in different situations and it seems to work quite well in the solar corona. Therefore, we think that the more realistic model of curved coronal tubes may significantly reduce the density contrast and remove the inconsistency. Corresponding calculations should be done in future.

The constancy of the Alfvén speed with height and the electron number densities at the heights of $0.1 R_{0}$ and $1 R_{0}$ $\left(4.67 \times 10^{8} \mathrm{~cm}^{-3}\right.$ and $4 \times 10^{6} \mathrm{~cm}^{-3}$ from gravitationally stratified corona) allows us to estimate the magnetic field strength at $0.1 R_{0}$ as $\sim 10 \mathrm{G}$. We note that early estimations of magnetic field strength in coronal loops at the height of $0.1-0.2 R_{0}$ are $300 \mathrm{G}$ by Asai et al. (2001), $13 \pm 9$ by Nakariakov \& Ofman (2001), 2-51 G by Wang et al. (2007), and $\sim 4 \mathrm{G}$ by Aschwanden \& Schrijver (2011). The magnetic field strength estimated from our observations agrees with the estimation of Nakariakov \& Ofman (2001).

The density structure of gravitationally stratified atmosphere with a temperature of $1 \mathrm{MK}$ has a scale height of $\sim 0.086 R_{0}$ near the coronal base. The constancy of the Alfvén speed with height gives the scale height of the magnetic field strength to be $\sim 0.17 R_{0}$. On the other hand, if one considers a current-free magnetic arcade, then the $z$-dependence of the magnetic field strength is proportional to $\exp \left(-z / \Lambda_{B}\right)$ with $\Lambda_{B}=l / \pi$, where $l$ is the horizontal extent of the arcade. For transequatorial loops one may consider $l$ as the distance between active regions in opposite hemispheres, i.e., in our case between AR 11190 and AR 11187, which is about $0.7 R_{0}$. This gives $\Lambda_{B} \approx 0.22 R_{0}$. Therefore, the seismologically estimated scale height is slightly different from the current-free magnetic configuration. This may indicate that the magnetic structure is not current-free and the current is caused by shearing and/or twisting of the arcade because of footpoint motions.

This is the first attempt to use radio observations for the estimation of plasma parameters in the outer solar corona $\left(>0.2 R_{0}\right)$. More observations and theoretical modeling are needed to test the estimations and to develop the seismology of outer solar corona.

\section{Conclusion}

We started using the radio observations to estimate the plasma parameters and to develop the radio seismology of the outer solar corona $\left(>0.2 R_{0}\right)$. As an example, we used the observations from the Ukrainian radio telescope URAN-2 on April 14, 2011. A type IV radio burst was detected in the frequency range of 13-32 MHz during the time interval of 09:50-12:30 UT, which is probably connected to a small C2.3 flare occurring in the active region AR 11190 between 09:38-09:49 UT. Wavelet analysis at four different frequencies $(29 \mathrm{MHz}, 25 \mathrm{MHz}, 22 \mathrm{MHz}$, and $14 \mathrm{MHz}$ ) shows the periodicity of $34 \mathrm{~min}$ and $23 \mathrm{~min}$ in the emission intensity. We suggest that the periodicity is caused by the first and second harmonics of vertical kink oscillations of the transequatorial loop system, which connects the active regions from the northern and southern hemispheres. Using the theoretical properties of kink oscillations in longitudinally inhomogeneous coronal loops, we estimate the Alfvén speed inside the loop at the height of $1 R_{0}$ to be $\sim 1000 \mathrm{~km} \mathrm{~s}^{-1}$. The coronal loop seems to be thin (with width to length ratio of 0.1 ), longitudinally weakly inhomogeneous in the Alfvén speed and dense $\left(\rho_{0} / \rho_{\mathrm{e}} \geq 20\right)$. The estimated magnetic field strength of the loop at the height of $1 R_{0}$ is $\sim 0.9 \mathrm{G}$. Extrapolation of this value gives the magnetic field strength at the height of $0.1 R_{0}$ as $\sim 10 \mathrm{G}$, which is in agreement with the estimation of Nakariakov \& Ofman (2001). More realistic calculations of MHD oscillations in a curved coronal tube with a longitudinal inhomogeneity may increase the accuracy of the estimations.

Acknowledgements. The work was supported by the European FP7-PEOPLE2010-IRSES-269299 project- SOLSPANET. The work of T.Z. was also supported by EU collaborative project STORM - 313038 and by Shota Rustaveli National Science Foundation grant DI/14/6-310/12. The work of M.P. was supported by the Austrian Fonds zur Förderung der wissenschaftlichen Forschung (project P23762-N16).

\section{Appendix A: Density stratification in the solar atmosphere}

Equilibrium pressure and density in a stratified atmosphere under gravity satisfy the equation

$\frac{\mathrm{d} p}{\mathrm{~d} R}=-\rho(R) \frac{G M}{R^{2}}$,

where $G=6.674 \times 10^{-8} \mathrm{~cm}^{3} \mathrm{~g}^{-1} \mathrm{~s}^{-2}$ is the gravitational constant, $M=1.989 \times 10^{33} \mathrm{~g}$ is the solar mass and $R$ is the distance from the solar center.

We may integrate Eq. (A.1) to obtain

$p(R)=p_{0}\left(R_{0}\right) \mathrm{e}^{-S_{0}(R)}, \rho(R)=\rho_{0}\left(R_{0}\right) \mathrm{e}^{-S_{0}(R)}$,

where $R_{0}$ is the solar radius,

$S_{0}(R)=\int_{R_{0}}^{R} \frac{\mathrm{d} z}{H_{0}(z)}$

is the integrated scale height and

$H_{0}(R)=\frac{p_{0}\left(R_{0}\right) R^{2}}{G M \rho_{0}\left(R_{0}\right)}$

is the pressure scale height. If we consider the equation of state $p=2 \mathrm{k} \rho T / \mathrm{m}$, where $k=1.38 \times 10^{-16} \mathrm{erg} \mathrm{K}^{-1}$ is the Boltzmann constant, $m=1.67 \times 10^{-24} \mathrm{~g}$ is the proton mass and $T=$ const. is the temperature, then we obtain from Eq. (A.3)

$S_{0}(R)=\frac{G M m}{2 k T R_{0}} \frac{R-R_{0}}{R}$.

Now considering $R=R_{0}+h$ we derive the following formula for the density

$\rho(h)=\rho_{0} \mathrm{e}^{-\frac{R_{0}}{H_{\mathrm{n}}} \frac{h}{R_{0}+h}}$,

where $H_{\mathrm{n}}=2 k T R_{0}^{2} / G M m$ is the pressure scale height near the solar surface. Then we obtain the equation for electron number density as

$n_{\mathrm{e}}(\zeta)=n_{\mathrm{e} 0} \mathrm{e}^{-\frac{R_{0}}{H_{\mathrm{n}}} \frac{\zeta}{1+\zeta}}$

where $\zeta=h / R_{0}$ is the normalized height above the solar surface.

\section{References}

Abramowitz, M., \& Stegun, I. A. 1964, Handbook of Mathematical Functions (Washington, D.C.: National Bureau of Standards)

Andries, J., Goossens, M., Hollweg, J. V., Arregui, I., \& Van Doorsselaere, T. 2005a, A\&A, 430, 1109

Andries, J., Arregui, I., \& Goossens, M. 2005b, ApJ, 624, L57

Andries, J., van Doorsselaere, T., Roberts, B., et al. 2009, Space Sci. Rev., 149, 3 
Arregui, I., \& Asensio Ramos, A. 2011, ApJ, 740, 44, 10

Arregui, I., Andries, J., Van Doorsselaere, T., Goossens, M., \& Poedts, S. 2007, A\&A, 463, 333

Arregui, I., Oliver, R., \& Ballester, J. L. 2012, Liv. Rev. Sol. Phys., 9, http: //www . livingreviews . org/lrsp-2012-2

Asai, A., Shimojo, M., Isobe, H., et al. 2001, ApJ, 562, L103

Aschwanden, M. J. 2004, Physics of the Solar Corona (Chichester/New York: Praxis/Springer)

Aschwanden, M. J., \& Schrijver, C. J. 2011, ApJ, 736, 102, 20

Aschwanden, M. J., Fletcher, L., Schrijver, C. J., \& Alexander, D. 1999, ApJ, 520,880

Aschwanden, M. J., Nakariakov, V. M., \& Melnikov, V. F. 2004, ApJ, 600, 458

Brady, C. S., \& Arber, T. D. 2005, A\&A, 438, 733

Brazhenko, A. I., Bulatsen, V. G., Vashchishin, R. V., et al. 2005, Kinematika i Fizika Nebesnykh Tel, 5, 43

Chase, R. C., Krieger, A. S., Svestka, Z., \& Vaiana, G. S. 1976, in Space Res. XVI (Berlin: Akademie), 917

De Moortel, I., \& Brady, C. S. 2007, A\&A, 664, 1210

Díaz, A. J., Oliver, R., Ballester, J. L., \& Roberts, B. 2004, A\&A, 424, 1055

Díaz, A. J., Zaqarashvili, T. V., \& Roberts, B. 2006, A\&A, 455, 709

Donnelly, G. R., Diáz, A., \& Roberts, B. 2006, A\&A, 457, 707

Dorovsky, V. V., Melnik, V. N., Konovalenko, A. A., et al. 2010, Radio Physics and Radio Astronomy, 1, 181

Dymova, M. V., \& Ruderman, M. S. 2006, A\&A, 457, 1059

Edwin, P. M., \& Roberts, B. 1983, Sol. Phys., 88, 179

Ginzburg, V. L., \& Zhelezniakov, V. V. 1958, Astron. Z., 35, 694

Gruszecki, M., Murawski, K., \& Ofman, L. 2008, A\&A, 488, 757

Khodachenko, M. L., Kislyakova, K. G., Zaqarashvili, T. V., et al. 2011, A\&A, 525, A105

Lemen, J. R., Title, A. M., Akin, D. J., et al. 2012, Sol. Phys., 275, 17

McEwan, M. P., Donnelly, G. R., Diaz, A. J., \& Roberts, B. 2006, A\&A, 460, 893

McEwan, M. P., Díaz, A. J., \& Roberts, B. 2008, A\&A, 481, 819

Megn, A. V., Sharykin, N. K., Zaharenko, V. V., et al. 2003, Radio Physics and Radio Astronomy, 8, 345

Melnik, V. N., Rucker, H. O., Konovalenko, A. A., et al. 2008, in Sol. Phys. Res. Trends, ed. Pingzhi Wang, (New York: Nova Science Publishers), 287

Melnik, V. N., Rucker, H. O., Konovalenko, A. A., et al. 2011, in Planetary Radio Emissions VII (Vienna: Austrian Academy of Sciences Press), 343

Nakariakov, V. M., \& Ofman, L. 2001, A\&A, 372, L53
Nakariakov, V. M., \& Verwichte, E. 2005, Liv. Rev. Sol. Phys., 2, http://www . livingreviews . org/lrsp-2005-3

Nakariakov, V. M., Ofman, L., Deluca, E. E., Roberts, B., \& Davila, J. M. 1999, Science, 285, 862

Nakariakov, V. M., Melnikov, V. F., \& Reznikova, V. M. 2003, A\&A, 412, L7

Ofman, L., Nakariakov, V. M., \& DeForest, C. E. 1999, ApJ, 514, 441

Pascoe, D. J., Nakariakov, V. M., Arber, T. D., \& Murawski, K. 2009, A\&A, 494, 1119

Pesnell, W. D., Thompson, B. J., \& Chamberlin, P. C. 2011, Sol. Phys., 275, 3

Pevtsov, A. A. 2000, ApJ, 531, 553

Roberts, B. 1981, Sol. Phys., 69, 39

Roberts, B., Edwin, P. M., \& Benz, A. O. 1984, ApJ, 279, 857

Ryabov, V. B., Vavriv, D. M., Zarka, P., et al. 2010, A\&A, 510, A16

Schou, J., Scherrer, P. H., Bush, R. I., et al. 2012, Sol. Phys., 275, 229

Selwa, M., Murawski, K., Solanki, S. K., Wang, T. J., \& Tóth, G. 2005, A\&A, 440,385

Selwa, M., Murawski, K., Solanki, S. K., \& Wang, T. J. 2007, A\&A, 462, 1127

Srivastava, A. K., Zaqarashvili, T. V., Uddin, W., Dwivedi, B. N., \& Kumar, P. 2008, MNRAS, 388, 1899

Sych, R., Zaqarashvili, T. V., Nakariakov, V. M., et al. 2012, A\&A, 539, A23

Terradas, J., Oliver, R., \& Ballester, J. L. 2006, ApJ, 642, 533

Van Doorsselaere, T., Debosscher, A., Andries, J., \& Poedts, S. 2004, A\&A, 424, 1065

Van Doorsselaere, T., Nakariakov, V. M., \& Verwichte, E. 2007, A\&A, 473, 959

Verth, G., \& Erdélyi, R. 2008, A\&A, 486, 1015

Verth, G., Erdélyi, R., \& Goossens, M. 2010, ApJ, 714, 1637

Verth, G., Goossens, M., \& He, J.-S. 2011, ApJ, 733, 15, L5

Verwichte, E., Nakariakov, V. M., Ofman, L., \& DeLuca, E. E. 2004, Sol. Phys., 223, 77

Verwichte, E., Foullon, C., \& Nakariakov, V. M. 2006, A\&A, 446, 1139

Wang, T. J., \& Solanki, S. K. 2004, A\&A, 421, L33

Wang, T. J., Solanki, S. K., Innes, D. E., et al. 2003a, A\&A, 402, L17

Wang, T. J., Solanki, S. K., Curdt, W., et al. 2003b, A\&A, 406, 1105

Wang, T. J., Innes, D. E., \& Qiu, J. 2007, ApJ, 656, 598

Zaqarashvili, T. V. 2003, A\&A, 399, L15

Zaqarashvili, T. V., \& Erdélyi, R. 2009, Space Sci. Rev., 149, 355

Zaqarashvili, T. V., \& Murawski, K. 2007, A\&A, 470, 353

Zaqarashvili, T. V., Khutsishvili, E., Kukhianidze, V., \& Ramishvili, G. 2007, A\&A, 474, 627 